\title{
Analysis of Mixed Elliptic and Parabolic Boundary Layers with Corners
}

\author{
Gung-Min Gie, ${ }^{1}$ Chang-Yeol Jung, ${ }^{2}$ and Roger Temam ${ }^{3}$ \\ ${ }^{1}$ Department of Mathematics, University of California, Riverside, 900 University Avenue, Riverside, CA 92521, USA \\ ${ }^{2}$ Ulsan National Institute of Science and Technology, San 194, Banyeon-ri, Eonyang-eup, Ulju Gun, Ulsan 689-798, Republic of Korea \\ ${ }^{3}$ The Institute for Scientific Computing and Applied Mathematics, Indiana University, 831 East Third Street, \\ Bloomington, IN 47405, USA
}

Correspondence should be addressed to Gung-Min Gie; ggie@math.ucr.edu

Received 27 January 2013; Accepted 1 April 2013

Academic Editor: Norio Yoshida

Copyright (C) 2013 Gung-Min Gie et al. This is an open access article distributed under the Creative Commons Attribution License, which permits unrestricted use, distribution, and reproduction in any medium, provided the original work is properly cited.

We study the asymptotic behavior at small diffusivity of the solutions, $u^{\varepsilon}$, to a convection-diffusion equation in a rectangular domain $\Omega$. The diffusive equation is supplemented with a Dirichlet boundary condition, which is smooth along the edges and continuous at the corners. To resolve the discrepancy, on $\partial \Omega$, between $u^{\varepsilon}$ and the corresponding limit solution, $u^{0}$, we propose asymptotic expansions of $u^{\varepsilon}$ at any arbitrary, but fixed, order. In order to manage some singular effects near the four corners of $\Omega$, the so-called elliptic and ordinary corner correctors are added in the asymptotic expansions as well as the parabolic and classical boundary layer functions. Then, performing the energy estimates on the difference of $u^{\varepsilon}$ and the proposed expansions, the validity of our asymptotic expansions is established in suitable Sobolev spaces.

\section{Introduction}

We consider a singularly perturbed convection-diffusion equation in a rectangular domain $\Omega=(0,1) \times(0,1)$ :

$$
\begin{gathered}
L_{\varepsilon} u^{\varepsilon}:=-\varepsilon \Delta u^{\varepsilon}-\partial_{x} u^{\varepsilon}=f \quad \text { in } \Omega, \\
u^{\varepsilon}=g \quad \text { on } \partial \Omega .
\end{gathered}
$$

Here $\varepsilon$ is a small but strictly positive diffusivity parameter, and $f=f(x, y)$ is a given smooth data with $\left\|D^{\alpha} f\right\|_{L^{2}(\Omega)} \leq$ $\kappa_{\alpha}$, independent of $\varepsilon$, for some $\alpha$ 's as needed in the analysis below. The function $g=g(x, y)$ is assumed to be continuous on $\partial \Omega$ and smooth on each edge of $\partial \Omega$. Namely, defining the restriction of $g$ to the edges of $\partial \Omega$ as follows:

$$
\begin{array}{ll}
\left.g\right|_{x=0}=g_{1}(y), & \left.g\right|_{x=1}=g_{2}(y), \\
\left.g\right|_{y=0}=g_{3}(x), & \left.g\right|_{y=1}=g_{4}(x),
\end{array}
$$

we assume that

$$
\begin{array}{ll}
g_{1}(0)=g_{3}(0), & g_{1}(1)=g_{4}(0), \\
g_{2}(0)=g_{3}(1), & g_{2}(1)=g_{4}(1),
\end{array}
$$

$g_{i}$ is smooth for $1 \leq i \leq 4$.

If these compatibility conditions were not satisfied, some additional considerations would be necessary, which we do not address here.

In what follows, we study the asymptotic behavior of the solutions to (1) at small diffusivity $\varepsilon$.

In a very nice related earlier work, [1], the asymptotic behavior of the solutions of a convection-diffusion equation similar to (1) was discussed. More precisely, in a rectangular domain $\widetilde{\Omega}=\left(0, l_{0}\right) \times\left(0, l_{1}\right)$, the authors considered

$$
\begin{aligned}
& -\varepsilon^{\prime} \Delta u^{\varepsilon^{\prime}}-b \partial_{x} u^{\varepsilon^{\prime}}+c u^{\varepsilon^{\prime}}=\widetilde{f} \text { in } \widetilde{\Omega}, \\
& u^{\varepsilon^{\prime}}=\widetilde{g} \quad \text { on } \partial \widetilde{\Omega} \text {, }
\end{aligned}
$$


where $b>0, c \geq 0$ are constants and $\tilde{f}$ is a given smooth function in $\widetilde{\Omega}$. The function $\widetilde{g}$, satisfying the analogue version of (3) in $\widetilde{\Omega}$, is assumed to be continuous and piecewise smooth on $\partial \widetilde{\Omega}$. By constructing, in [1], asymptotic expansions of $\mathcal{u}^{\varepsilon^{\prime}}$ with respect to a small diffusivity $\varepsilon^{\prime}$, the boundary layers of (4) were analyzed in a systematic way. The validity of their asymptotic expansions was established using the maximum principle.

Using a simple change of variables which maps $\widetilde{\Omega}$ to $\Omega$, and setting $u^{\varepsilon^{\prime}}=u^{\varepsilon} e^{\lambda x}$ with a suitable $\lambda$, one can transform (4) to (1) where the two diffusivity parameters $\varepsilon$ and $\varepsilon^{\prime}$, respectively, in (1) and (4) are compatible; that is, $\varepsilon / \varepsilon^{\prime}$ is of order one. Hence, via this transformation, our analysis of (1) in this paper is applicable to (4) as well. Our motivations for conducting the present study appear below. In particular we significantly simplify the proofs of [1] and make the study suitable for more general equations or systems, in particular, those which do not satisfy the maximum principle.

In the boundary layer analysis associated with the convection-diffusion equation (1) (or (4)), one of the most important and difficult points is to resolve any possible singularity near the four corners of the rectangular domain $\Omega$. Towards this end, we simplify and improve the method used in [1]. More precisely, concerning the asymptotic expansion of $u^{\varepsilon}$, we introduce the so-called elliptic boundary layer corrector, appearing in Section 3.2, near the inflow boundary at $x=1$ only, while in [1], it was used near both the inflow and outflow boundaries at $x=1$ and $x=0$. This simplification in the construction of the asymptotic expansions is mainly based on an observation that the corner singularities at $(0,0)$ and $(0,1)$ (where the flows go out) can be handled by the ordinary corner corrector, appearing in Section 3.4 (which is much easier to analyze than the elliptic corrector). Using the energy estimates rather than the maximum principle as in [1], we prove the validity of our proposed asymptotic expansions in suitable Sobolev spaces. Here we make use of the Hardy inequality (see, e.g., [2]) in the estimates. As we said above, our energy estimation approach can be easily extended to some higher order equations or systems which do not admit a maximum principle.

This paper is organized as follows. In Section 2, we introduce a formal expansion of $u^{\varepsilon}$ as the sum of the outer and inner expansions. The outer expansion (outside of the boundary layers), which is easy to obtain, appears in Section 2. Then, in Section 3, by performing the boundary layer analysis in a systematic way, we construct the inner expansion (inside the boundary layers). In Section 4, we state and prove the main convergence result, Theorem 9, concerning the difference between $u^{\varepsilon}$ and the proposed asymptotic expansion at a given order. In addition, in the appendix, we prove Lemma 1 that contains some delicate pointwise estimates on the parabolic boundary layer correctors introduced in Section 3.1. The study of the elliptic corrector and its approximation also appears in the appendix. below.
Notation 1. $\kappa:=\kappa(f, g, \Omega, n)$ is a constant depending on the data, but independent of $\varepsilon$; here $n \geq 0$ is the fixed (but arbitrary) order of the asymptotic expansion, as it appears in (6).

Notation 2. An e.s.t. is a function (or a constant) whose norm in all Sobolev spaces $H^{s}$ (and thus spaces $C^{s}$ ) is exponentially small with, for each $s$, a bound of the form $c_{1} e^{-c_{2} / \varepsilon^{\gamma}}, c_{1}, c_{2}, \gamma>$ $0, c_{i}, \gamma$ depending possibly on $s$.

Notation 3. For a fixed $\varepsilon>0$, we define the energy norm of $H^{1}(\Omega)$,

$$
\|\cdot\|_{\varepsilon}:=\sqrt{\varepsilon}\|\cdot\|_{H^{1}(\Omega)}+\|\cdot\|_{L^{2}(\Omega)} \cdot
$$

\section{Asymptotic Expansions}

To study the asymptotic behavior of $u^{\varepsilon}$, solution of (1), we propose an asymptotic expansion $u^{\varepsilon}$ of the following type:

$$
u^{\varepsilon} \cong \sum_{j=0}^{n} \varepsilon^{j}\left(u^{j}+\Theta^{j}\right)
$$

Here, at each order of $\varepsilon^{j}, j \geq 0, u^{j}$ corresponds to the outer expansion (outside of the boundary layer) of $u^{\varepsilon}$. To balance the discrepancy on the boundary $\partial \Omega$ of $u^{\varepsilon}$ and of the $u^{j}, j \geq 0$, we introduce the correctors $\Theta^{j}, j \geq 0$, which will contribute mainly inside of the boundary layer: $\Theta^{j}$ will be itself the sum of several boundary layer functions as we will see below. The asymptotic expansion (6) is made at order $n \geq 0$. As we will see below, the expansion itself depends on $n$, but $n$, which is set at the beginning of the study, can be chosen arbitrary large.

To determine the asymptotic expansion (6), we start with the outer expansion for $u^{\varepsilon}, u^{\varepsilon} \cong \sum_{j=0}^{n} \varepsilon^{j} u^{j}$. Inserting this expansion into (1), we formally find the equations for the $u^{j}$ :

$$
\begin{gathered}
-\partial_{x} u^{0}=f, \\
-\partial_{x} u^{j}=\Delta u^{j-1}, \quad 1 \leq j \leq n .
\end{gathered}
$$

We supplement these equations with the following inflow boundary conditions (see (1)-(3)):

$$
\begin{gathered}
u^{0}=g_{2}(y) \quad \text { at } x=1, \\
u^{j}=0 \quad \text { at } x=1,1 \leq j \leq n .
\end{gathered}
$$

While (7) are "natural", the choice of the boundary conditions in (8) is not obvious; it will be eventually justified by the convergence theorem below. Then, by integrating the equations in $x$, we find the smooth outer solutions $u^{j}$ in the following form:

$$
\begin{gathered}
u^{0}=\int_{x}^{1} f\left(x_{1}, y\right) d x_{1}+g_{2}(y), \\
u^{j}=\int_{x}^{1} \Delta u^{j-1}\left(x_{1}, y\right) d x_{1}, \quad 1 \leq j \leq n .
\end{gathered}
$$


Under this construction of the outer expansion, we notice that $u^{\varepsilon} \cong \sum_{j=0}^{n} \varepsilon^{j} \mathcal{u}^{j}$ satisfies the boundary condition $(1)_{2}$ along the edge $x=1$ only, and not on the three other sides of $\partial \Omega, y=0, y=1$, and $x=0$ (in general). Hence, we expect boundary layers to occur near those edges, and the socalled correctors (corresponding to the $\Theta^{j}$ ) will be necessary to account for these discrepancies.

In Section 3, performing the boundary layer analysis, we define, for each $0 \leq j \leq n$, the corrector $\Theta^{j}$ appearing in (11) below. Then, in Section 4, using (6), (9), (10), and (11), we prove our main result which is stated as Theorem 9 .

\section{Construction of the Correctors: Boundary Layers Analysis}

We want the boundary value of $u^{\varepsilon}$ to match that of its approximation. In general, the boundary values of the diffusive solution $\mathcal{u}^{\varepsilon}$ and the outer expansion $\sum_{j=0}^{n} \varepsilon^{j} \mathcal{u}^{j}$ match only at $x=1$, but not on the other sides of $\partial \Omega, y=0, y=1$, and $x=0$. Hence, we expect that some boundary layers will occur near those three edges. To resolve this inconsistency, we will define a number of boundary layer functions on the sides and corners of $\partial \Omega$, following an analysis reminiscent of the theory of the Prandtl boundary layer in [3-6].

For the moment, comparing $u^{\varepsilon}$ with a finite sum $\sum_{j=0}^{n} \varepsilon^{j} u^{j}$, we infer from the definition of the $u^{j}$ in (7)-(10) that the values of these functions coincide on the segment $x=1$. In general, they will not coincide anywhere else on $\partial \Omega$. First, to correct the inconsistency on the sides $y=0,1$, we will define the so-called parabolic correctors $\varphi^{j}=\varphi_{l}^{j}+$ $\varphi_{u}^{j}$. Then, to correct the inconsistency at $x=0$, we will introduce the (classical) corrector $\theta^{j}$. However, this will not be enough because additional inconsistencies appear at the corners of $\Omega$, and, as we shall see below, it will be necessary to introduce additional correctors $\xi^{j}, \zeta^{j}$, and $\eta^{j}$ to handle these inconsistencies.

In summary, in the subsections below, we will define the $\Theta^{j}$ in the form,

$$
\Theta^{j}=\varphi^{j}+\xi^{j}+\theta^{j}+\zeta^{j}+\eta^{j}, \quad 0 \leq j \leq n,
$$

where the role and location of each corrector are explained, respectively, in Box 1 and Figure 1. The stretched variables of the corrector functions, which will be introduced and used in the following subsections, are indicated in the following equation:

$$
\begin{gathered}
\varphi_{l}^{j}=\varphi_{l}^{j}\left(x, \frac{y}{\sqrt{\varepsilon}}\right), \quad \varphi_{u}^{j}=\varphi_{u}^{j}\left(x, \frac{(1-y)}{\sqrt{\varepsilon}}\right), \\
\xi_{l}^{j}=\xi_{l}^{j}\left(\frac{(1-x)}{\varepsilon}, \frac{y}{\varepsilon}\right), \quad \xi_{u}^{j}=\xi_{u}^{j}\left(\frac{(1-x)}{\varepsilon}, \frac{(1-y)}{\varepsilon}\right), \\
\theta^{j}=\theta^{j}\left(\frac{x}{\varepsilon}, y\right),
\end{gathered}
$$

$$
\begin{array}{cc}
\zeta_{l}^{j}=\zeta_{l}^{j}\left(\frac{x}{\varepsilon}, \frac{y}{\sqrt{\varepsilon}}\right), \quad \zeta_{u}^{j}=\zeta_{u}^{j}\left(\frac{x}{\varepsilon}, \frac{(1-y)}{\varepsilon}\right), \\
\eta_{l}^{j}=\eta_{l}^{j}\left(\frac{x}{\varepsilon}, y\right), \quad \eta_{u}^{j}=\eta_{u}^{j}\left(\frac{x}{\varepsilon}, y\right) .
\end{array}
$$

3.1. Parabolic Boundary Layers (PBL). At the bottom (or top) boundary, that is, at $y=0$ (or $y=1$ ), to balance the discrepancy of $u^{\varepsilon}$ and the outer expansion $\sum_{j=0}^{n} \varepsilon^{j} u^{j}$, (see the $u^{j}$ as defined in (9) and (10)), we construct below the parabolic boundary layer correctors $\varphi^{j}, 0 \leq j \leq n$.

We formally insert the asymptotic expansion $u^{\varepsilon} \cong$ $\sum_{j=0}^{n} \varepsilon^{j} \varphi^{j}$ into (1). Using the arguments similar to those of the Prandtl theory, we see that the thickness of the boundary layer near $y=0$ or $y=1$ should be of order $\varepsilon^{1 / 2}$. Then, by collecting the terms at the same order of $\varepsilon^{j}, 0 \leq j \leq n$, we find the equations of the parabolic boundary layer correctors:

$$
-\varepsilon \partial_{y}^{2} \varphi^{j}-\partial_{x} \varphi^{j}=\partial_{x}^{2} \varphi^{j-1} \quad \text { in } \Omega, 0 \leq j \leq n,
$$

where we set $\varphi^{-1}=0$.

Along the boundaries $y=0, y=1$, and $x=1$, comparing $(1)_{2}$ and the boundary values of the functions in (9) and (10), we find the boundary conditions of $\varphi^{j}, 0 \leq j \leq n$, which read.

$$
\begin{gathered}
\varphi^{j}=0 \quad \text { at } x=1,0 \leq j \leq n, \\
\varphi^{0}=g_{3}(x)-u^{0}(x, 0) \quad \text { at } y=0, \\
\varphi^{j}=-u^{j}(x, 0) \quad \text { at } y=0,1 \leq j \leq n, \\
\varphi^{0}=g_{4}(x)-u^{0}(x, 1) \quad \text { at } y=1, \\
\varphi^{j}=-u^{j}(x, 1) \quad \text { at } y=1,1 \leq j \leq n .
\end{gathered}
$$

We will show below how to actually construct $\varphi^{j}$. For the moment, comparing the functions $u^{\varepsilon}$ and $\sum_{j=0}^{n} \varepsilon^{j}\left(u^{j}-\varphi^{j}\right)$ for some $n \geq 0$, we see that the boundary values of these functions coincide at $x=1$ and $y=0,1$. However this has been achieved at the price of introducing some new inconsistencies. Indeed we notice, in (14), that, for each $j \geq 0$, the boundary conditions at $y=0$ and $y=1$ are not consistent with that at $x=1$. That is, in general, for any $i \geq 1$,

$$
\begin{gathered}
\partial_{x}^{i} u^{0}(1,0)-\partial_{x}^{i} g_{3}(1) \neq 0, \quad \partial_{x}^{i} u^{0}(1,1)-\partial_{x}^{i} g_{4}(1) \neq 0, \\
\partial_{x}^{i} u^{j}(1,0) \neq 0, \quad \partial_{x}^{i} u^{j}(1,1) \neq 0, \quad j \geq 1 .
\end{gathered}
$$

Due to this inconsistency of the boundary data, if we define $\varphi^{j}, 0 \leq j \leq n$, as a solution of (13) with (14), some derivatives of $\varphi^{j}, 0 \leq j \leq n$ get singular at the two corners $(1, k), k=0,1$. Therefore, to define smooth (smoother) correctors $\varphi^{j}, 0 \leq j \leq$ $n$, we will modify the boundary conditions (14) as introduced in (23). 
$\varphi^{j}=\varphi_{1}^{j}+\varphi_{u}^{j}$ is the parabolic boundary layer corrector near $y=0$ and $y=1$,

$\xi^{j}=\xi_{l}^{j}+\xi_{u}^{j}$ is the elliptic corrector which resolves the compatibility issues, in the construction of $\varphi^{j}$, at the corners $(1,0)$ and $(1,1)$,

$\theta^{j}$ is the ordinary boundary layer corrector near $x=0$,

$\zeta^{j}=\zeta_{l}^{j}+\zeta_{u}^{j}$ is the ordinary corner layer corrector which manages the effect of $\varphi^{j}$ along $x=0$,

$\eta^{j}=\eta_{l}^{j}+\eta_{u}^{j}$ is the supplementary corrector that manages the effects of $\theta^{j}$ and $\zeta^{j}$ along the edges $y=0$ and $y=1$.

Box 1: The different correctors.

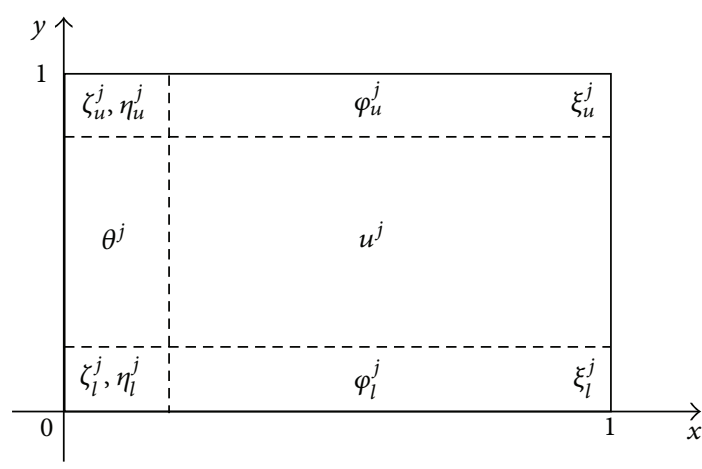

FIGURE 1: Location of the outer solution $u^{j}$ and the boundary layer correctors.

3.1.1. On Modification of Boundary Conditions for Smooth Correctors at $y=0,1$. Equation (13) with initial and boundary conditions (14) is a parabolic problem (heat equation), in which $-x$ is the (positive) time like variable so that the boundary conditions at $x=1$ are the analogue of the initial conditions for an evolution problem, and the possible inconsistencies in (15) correspond to the (analogue of ) the compatibility conditions between the initial and boundary values for a parabolic equation; see [7-9] and the references therein. Due to the lack of consistency at the corners $(1,0)$ and $(1,1)$, the correctors constructed as in (13) and (14) might get singular derivatives at those corners. We will overcome this difficulty by considering some corner functions at $x=1$ and $y=0,1$, similar to what is done in $[10,11]$ or $[12]$ for the compatibility issue in parabolic problems; see [13] as well.

Defining $\delta(x)$ as a smooth cut-off function, independent of $\varepsilon$, such that

$$
\delta(x)= \begin{cases}0 & \text { for } 0 \leq x \leq \frac{1}{2} \\ 1 & \text { for } x \geq \frac{3}{4}\end{cases}
$$

we set

$$
\gamma_{k}^{j}(x)=\tilde{\gamma}_{k}^{j}(x) \delta(x), \quad k=0,1
$$

where

$$
\begin{aligned}
& \widetilde{\gamma}_{k}^{0}(x)=\sum_{i=1}^{2 n+1} \frac{(x-1)^{i}}{i !}\left[\partial_{x}^{i} g_{3+k}(1)-\partial_{x}^{i} u^{0}(1, k)\right], \\
& \tilde{\gamma}_{k}^{j}(x)=-\sum_{i=1}^{2 n+1-2 j} \frac{(x-1)^{i}}{i !} \partial_{x}^{i} u^{j}(1, k), \quad 1 \leq j \leq n .
\end{aligned}
$$

Note that the $\widetilde{\gamma}_{k}^{0}, k=0,1$, depend on $n$, but this dependency is not made explicit to make the notations (slightly) simpler. Note also (comparing to (15)) that, at $x=1$, for each $k=0,1$,

$$
\begin{gathered}
\tilde{\gamma}_{k}^{j}(1)=0, \quad 0 \leq j \leq n, \\
\partial_{x}^{i} \widetilde{\gamma}_{k}^{0}(1)=\partial_{x}^{i} g_{3+k}(1)-\partial_{x}^{i} u^{0}(1, k), \quad 1 \leq i \leq 2 n+1, \\
\partial_{x}^{i} \widetilde{\gamma}_{k}^{j}(1)=-\partial_{x}^{i} u^{j}(1, k), \quad 1 \leq j \leq n, \quad 1 \leq i \leq 2 n+1-j .
\end{gathered}
$$

We introduce the stretched variables,

$$
\bar{y}=\frac{y}{\sqrt{\varepsilon}}, \quad \tilde{y}=\frac{(1-y)}{\sqrt{\varepsilon}} .
$$

Then, using (13), (14), (17), and (20), we define the parabolic corrector, $\varphi^{j}, 0 \leq j \leq n$, as

$$
\varphi^{j}=\varphi_{l}^{j}+\varphi_{u}^{j}=\varphi_{l}^{j}(x, \bar{y})+\varphi_{u}^{j}(x, \widetilde{y}),
$$

where $\varphi_{l}^{j}$ and $\varphi_{u}^{j}$ (lower and upper parts of $\varphi^{j}$, resp.) are defined as the solutions of the 1D heat equations below.

For $0 \leq j \leq n$,

$$
\begin{aligned}
&-\partial_{\bar{y}}^{2} \varphi_{l}^{j}-\partial_{x} \varphi_{l}^{j}=\partial_{x}^{2} \varphi_{l}^{j-1} \quad \text { for }(\bar{y}, x) \in \mathbb{R}^{+} \times(0,1), \text { at } \bar{y}=0, \\
& \varphi_{l}^{j}=h_{0}^{j}(x) \quad \text { as } \bar{y} \longrightarrow \infty, \\
& \varphi_{l}^{j} \longrightarrow 0 \quad \text { at } x=1, \\
& \varphi_{l}^{j}=0 \quad \text { for }(\tilde{y}, x) \in \mathbb{R}^{+} \times(0,1), \\
&-\partial_{\tilde{y}}^{2} \varphi_{u}^{j}-\partial_{x} \varphi_{u}^{j}=\partial_{x}^{2} \varphi_{u}^{j-1} \quad \text { at } \tilde{y}=0, \\
& \varphi_{u}^{j}=h_{1}^{j}(x) \quad \text { as } \tilde{y} \longrightarrow \infty, \\
& \varphi_{u}^{j} \longrightarrow 0 \quad \text { at } x=1 .
\end{aligned}
$$

Here the $h_{k}^{j}(x), 0 \leq j \leq n, k=0,1$, are defined by

$$
\begin{gathered}
h_{0}^{0}(x):=g_{3}(x)-u^{0}(x, 0)-\gamma_{0}^{0}(x), \\
h_{0}^{j}(x):=-u^{j}(x, 0)-\gamma_{0}^{j}(x), \quad 1 \leq j \leq n, \\
h_{1}^{0}(x):=g_{4}(x)-u^{0}(x, 1)-\gamma_{1}^{0}(x), \\
h_{1}^{j}(x):=-u^{j}(x, 1)-\gamma_{1}^{j}(x), \quad 1 \leq j \leq n .
\end{gathered}
$$


Note that the smooth boundary conditions $h_{k}^{j}(x), 0 \leq j \leq n$, $k=0,1$, along $\bar{y}$ or $\tilde{y}=0$ are compatible with the other 0 boundary (initial) conditions at $x=1$ in the sense that, for each $0 \leq j \leq n$,

$$
\partial_{x}^{i} h_{k}^{j}(1)=0, \quad k=0,1,0 \leq i \leq 2 n+1-2 j .
$$

From [14-16] or [1], we recall the explicit expressions of $\varphi_{l}^{j}=\varphi_{l}^{j}(x, \bar{y}), 0 \leq j \leq n$,

$$
\begin{array}{r}
\varphi_{l}^{0}=\sqrt{\frac{2}{\pi}} \int_{\bar{y} / \sqrt{2(1-x)}}^{\infty} \exp \left(-\frac{y_{1}^{2}}{2}\right) h_{0}^{0}\left(x+\frac{\bar{y}^{2}}{2 y_{1}^{2}}\right) d y_{1}, \quad(25) \\
\varphi_{l}^{j}=\sqrt{\frac{2}{\pi}} \int_{\bar{y} / \sqrt{2(1-x)}}^{\infty} \exp \left(-\frac{y_{1}^{2}}{2}\right) h_{0}^{j}\left(x+\frac{\bar{y}^{2}}{2 y_{1}^{2}}\right) d y_{1} \\
+\frac{1}{2 \sqrt{\pi}} \int_{0}^{1-x} \int_{0}^{\infty} \frac{1}{\sqrt{x_{1}}}\left\{\exp \left(-\frac{\left(\bar{y}-y_{1}\right)^{2}}{4 x_{1}}\right)\right. \\
\times \partial_{x}^{2} \varphi_{l}^{j-1}\left(x+x_{1}, y_{1}\right) d y_{1} d x_{1} .
\end{array}
$$

The expressions of $\varphi_{u}^{j}=\varphi_{u}^{j}(x, \widetilde{y}), 0 \leq j \leq n$, are identical to (25) and (26) with $h_{0}^{0}, h_{0}^{j}$, and $\bar{y}$, respectively, replaced by $h_{1}^{0}, h_{1}^{j}$ and $\tilde{y}$.

3.1.2. Estimates on the Parabolic Boundary Layers. We now state some pointwise estimates for the $\varphi_{l}^{j}$ and $\varphi_{u}^{j}, 0 \leq j \leq n$, which are proved in the appendix.

Lemma 1. For each $0 \leq j \leq n$ and $s \geq 0$, one has the following pointwise estimates:

$$
\begin{array}{r}
\left|y^{s} \partial_{x}^{i} \partial_{y}^{m} \varphi_{l}^{j}\right| \leq \kappa \varepsilon^{(s-m) / 2} \exp \left(-c \frac{y}{\sqrt{\varepsilon}}\right), \\
0 \leq i+m \leq 2 n+2-2 j, \\
\left|y^{s} \partial_{x}^{i} \partial_{y}^{m} \varphi_{u}^{j}\right| \leq \kappa \varepsilon^{(s-m) / 2} \exp \left(-c \frac{1-y}{\sqrt{\varepsilon}}\right), \\
0 \leq i+m \leq 2 n+2-2 j,
\end{array}
$$

for a generic constant $c>0$ independent of $x, y$, and $\varepsilon$.

From Lemma 1 , it is easy to deduce the following $L^{p}$ estimates.

Lemma 2. For each $0 \leq j \leq n$ and $s \geq 0$, one has, for $0 \leq$ $i+m \leq 2 n+2-2 j$,

$$
\left\|y^{s} \partial_{x}^{i} \partial_{y}^{m} \varphi_{l}^{j}\right\|_{L^{p}(\Omega)}+\left\|y^{s} \partial_{x}^{i} \partial_{y}^{m} \varphi_{u}^{j}\right\|_{L^{p}(\Omega)} \kappa \varepsilon^{(s-m) / 2+1 / 2 p}
$$

Using (21) and (22), we find the equations for $\varphi^{j}=\varphi_{l}^{j}+\varphi_{u}^{j}$, $0 \leq j \leq n$ :

$$
\begin{gathered}
-\varepsilon \partial_{y}^{2} \varphi^{j}-\partial_{x} \varphi^{j}=\partial_{x}^{2} \varphi^{j-1} \quad \text { in } \Omega, \\
\varphi^{j}=h_{0}^{j}(x)+\left.\varphi_{u}^{j}\right|_{y=0} \text { at } y=0, \\
\varphi^{j}=h_{1}^{j}(x)+\left.\varphi_{l}^{j}\right|_{y=1} \text { at } y=1, \\
\varphi^{j}=0 \quad \text { at } x=1 .
\end{gathered}
$$

Thanks to Lemma 1, we notice that

$$
\left.\varphi_{u}^{j}\right|_{y=0}=\text { e.s.t., }\left.\quad \varphi_{l}^{j}\right|_{y=1}=\text { e.s.t., }
$$

where e.s.t. denotes an exponentially small term with respect to the (small) parameter $\varepsilon$ as defined in Notation 2.

3.2. Elliptic Boundary Layers (EBL). In Section 3.1, to construct the consistent parabolic boundary layer correctors $\varphi^{j}$, $0 \leq j \leq n$, we considered the modified boundary conditions (23), including $\gamma_{k}^{j}$, instead of the natural boundary conditions (14). Here, on the two sides $y=0$ and $y=1$ of $\Omega$, to cancel $\gamma_{k}^{j}$ which is exactly the difference of (14) and (23), we introduce elliptic boundary layer correctors $\xi^{j}, 0 \leq j \leq n$, which, like $\gamma_{k}^{j}$ or $\widetilde{\gamma}_{k}^{j}$, depend on the order $n$ of the asymptotic expansion in (6).

Using (17), we define

$$
\xi^{j}=\xi_{l}^{j}+\xi_{u}^{j}, \quad 0 \leq j \leq n,
$$

where $\xi_{l}^{j}$ and $\xi_{u}^{j}$ are the solutions of the elliptic systems below.

For $0 \leq j \leq n$,

$$
\begin{gathered}
-\varepsilon \partial_{x}^{2} \xi_{l}^{j}-\varepsilon \partial_{y}^{2} \xi_{l}^{j}-\partial_{x} \xi_{l}^{j}=0 \quad \text { in } \Omega, \\
\xi_{l}^{j}=\gamma_{0}^{j}(x) \quad \text { at } y=0, \\
\xi_{l}^{j}=0 \quad \text { at } x=0,1, \text { or } y=1, \\
-\varepsilon \partial_{x}^{2} \xi_{u}^{j}-\varepsilon \partial_{y}^{2} \xi_{u}^{j}-\partial_{x} \xi_{u}^{j}=0 \quad \text { in } \Omega, \\
\xi_{u}^{j}=\gamma_{1}^{j}(x) \quad \text { at } y=1, \\
\xi_{u}^{j}=0 \quad \text { at } x=0,1, \text { or } y=0 .
\end{gathered}
$$

The systems (33) being elliptic equations, their wellposedness are easy to verify, and hence, we omit the proof here.

The equations satisfied by the elliptic correctors $\xi^{j}=\xi_{l}^{j}+$ $\xi_{u}^{j}$ read as follows.

For $0 \leq j \leq n$,

$$
\begin{gathered}
-\varepsilon \partial_{x}^{2} \xi^{j}-\varepsilon \partial_{y}^{2} \xi^{j}-\partial_{x} \xi^{j}=0 \quad \text { in } \Omega, \\
\xi^{j}=\gamma_{k}^{j}(x) \quad \text { at } y=k, k=0,1, \\
\xi^{j}=0 \quad \text { at } x=0,1 .
\end{gathered}
$$


In the error analysis below in Section 4, we will see that no extra terms related to the $\xi^{j}$ appear; see (65) below. This is because the $\xi^{j}$, as appearing in (34), satisfies the same equation as for $u^{\varepsilon}$, supplemented with the (exact) boundary conditions that we need. From this observation, as it will be justified in Section 4, we notice that our main result in Theorem 9 does not require any estimate on the $\xi^{j}$.

As an extra information which might be useful elsewhere, but not in this paper, by performing the energy estimate on (34), we find that

$$
\left\|\xi^{j}\right\|_{\varepsilon} \leq \kappa \varepsilon^{1 / 4}, \quad 0 \leq j \leq n
$$

In the appendix, we introduce an explicit approximate $\bar{\xi}$ of $\xi$. Then, thanks to the estimates on $\bar{\xi}$, one can obtain some pointwise estimates on $\xi$ as well; see Appendix B.

At this stage, the functions $u^{\varepsilon}$ and $\sum_{j=0}^{n} \varepsilon^{j}\left(u^{j}+\varphi^{j}+\xi^{j}\right)$ match along the sides $x=1$ and $y=0,1$ (with singular derivatives of high orders for the $\left.\varphi^{j}\right)$. More precisely, from (1), (8), (30), (31), and (34), we see that

$$
u^{\varepsilon}-\sum_{j=0}^{n} \varepsilon^{j}\left(u^{j}+\varphi^{j}+\xi^{j}\right)= \begin{cases}g_{1}+\sum_{j=0}^{n} \varepsilon^{j}\left(u^{j}+\varphi^{j}\right) & \text { at } x=0, \\ 0 & \text { at } x=1, \\ \sum_{j=0}^{n} \varepsilon^{j} \varphi_{u}^{j}=\text { e.s.t. } & \text { at } y=0, \\ \sum_{j=0}^{n} \varepsilon^{j} \varphi_{l}^{j}=\text { e.s.t. } & \text { at } y=1 .\end{cases}
$$

We will now deal with the side $x=0$ and construct the ordinary boundary layer $\theta^{j}$ and the ordinary corner layer functions (correctors) $\zeta^{j}$.

3.3. Ordinary Boundary Layers $(O B L)$. To handle the discrepancy of $u^{\varepsilon}$ and $\sum_{j=0}^{n} \varepsilon^{j} u^{j}$ at $x=0$, which is a part of $u^{\varepsilon}-\sum_{j=0}^{n} \varepsilon^{j}\left(u^{j}+\varphi^{j}+\xi^{j}\right)$ appearing in (36), the so-called ordinary boundary layer corrector $\theta^{j}$ is introduced in this section. We insert a formal expansion $u^{\varepsilon} \cong \sum_{j=0}^{\infty} \varepsilon^{j} \theta^{j}$ into the diffusive equation (1). Then, using the stretched variable $\bar{x}=x / \varepsilon$, we find the equations of $\theta^{j}$ :

$$
-\varepsilon \partial_{x}^{2} \theta^{j}-\partial_{x} \theta^{j}=\varepsilon^{-1} \partial_{y}^{2} \theta^{j-2} \quad \text { in } \Omega, 0 \leq j \leq n,
$$

where we set $\theta^{-1}=\theta^{-2}=0$.

The main role of the $\theta^{j}$ is, at each order of $\varepsilon^{j}$, to cancel the error $u^{\varepsilon}-\sum_{j=0}^{n} \varepsilon^{j} u^{j}$ at $x=0$. Hence, we supplement (37) with the boundary conditions

$$
\begin{gathered}
\theta^{0}=g_{1}(y)-u^{0}(0, y) \quad \text { at } x=0, \\
\theta^{j}=-u^{j}(0, y) \quad \text { at } x=0,1 \leq j \leq n, \\
\theta^{j} \longrightarrow 0 \text { as } x \longrightarrow \infty, 0 \leq j \leq n .
\end{gathered}
$$

The explicit expressions of $\theta^{j}, 0 \leq j \leq n$, are inductively shown to be of the form

$$
\theta^{j}=P^{k}\left(\frac{x}{\varepsilon}, y\right) \exp \left(\frac{-x}{\varepsilon}\right), \quad j=2 k, 2 k+1,
$$

where $P^{k}(x / \varepsilon, y)$ is a polynomial in $x / \varepsilon$ of degree $k$ whose coefficients, independent of $\varepsilon$, are linear combinations of the $\partial^{s} u^{j-s}(0, y) / \partial y^{s}, 0 \leq s \leq j$, and $P^{k}(0, y)=-u^{j}(0, y)$.

According to (39), we deduce the following lemmas.

Lemma 3. For each $0 \leq j \leq n$, one has the pointwise estimates:

$$
\left|x^{s} \partial_{x}^{i} \partial_{y}^{m} \theta^{j}\right| \leq \kappa \varepsilon^{s-i} \exp \left(-c \frac{x}{\varepsilon}\right), \quad s, i, m \geq 0
$$

for a constant $c$ independent of $x, y$, and $\varepsilon$.

Lemma 4. For each $0 \leq j \leq n$, one has

$$
\left\|x^{s} \partial_{x}^{i} \partial_{y}^{m} \theta^{j}\right\|_{L^{p}(\Omega)} \leq \kappa \varepsilon^{s-i+(1 / p)}, \quad s, i, m \geq 0 .
$$

Thanks to Lemma 3, we notice that the effect of $\theta^{j}$ near the boundary $x=1$ is exponentially small:

$$
\theta^{j}(1, y)=\text { e.s.t., } \quad 0 \leq j \leq n .
$$

From (36), (38), and (42), we infer that

$$
\begin{aligned}
u^{\varepsilon}-\sum_{j=0}^{n} \varepsilon^{j}\left(u^{j}+\varphi^{j}+\xi^{j}+\theta^{j}\right) & \\
& = \begin{cases}\sum_{j=0}^{n} \varepsilon^{j} \varphi^{j} & \text { at } x=0, \\
\sum_{j=0}^{n} \varepsilon^{j} \theta^{j}=\text { e.s.t. } & \text { at } x=1, \\
\sum_{j=0}^{n} \varepsilon^{j}\left(\varphi_{u}^{j}+\theta^{j}\right)=\sum_{j=0}^{n} \varepsilon^{j} \theta^{j}+\text { e.s.t. } & \text { at } y=0, \\
\sum_{j=0}^{n} \varepsilon^{j}\left(\varphi_{l}^{j}+\theta^{j}\right)=\sum_{j=0}^{n} \varepsilon^{j} \theta^{j}+\text { e.s.t. } & \text { at } y=1 .\end{cases}
\end{aligned}
$$

3.4. Ordinary Corner Layers (OCL). To account for the value $\sum_{j=0}^{n} \varepsilon^{j} \varphi^{j}$ at $x=0$, which is exactly the difference of $u^{\varepsilon}$ and $\sum_{j=0}^{n} \varepsilon^{j}\left(u^{j}+\varphi^{j}+\xi^{j}+\theta^{j}\right)$ at $x=0$ (see (43)), we introduce the ordinary corner layer correctors $\zeta^{j}, 0 \leq j \leq n$, in the form

$$
\zeta^{j}=\zeta_{l}^{j}+\zeta_{u}^{j}
$$

where the $\zeta_{l}^{j}$ (or $\left.\zeta_{u}^{j}\right)$ are the correctors near the corner $(0,0)$ (or $(0,1))$ as constructed below.

To define $\zeta_{l}^{j}$ (or $\zeta_{u}^{j}$ ), we insert a formal expansion $u^{\varepsilon} \cong$ $\sum_{j=0}^{\infty} \varepsilon^{j} \zeta^{j}$ into (1). Then, using the stretched variables $\bar{x}=x / \varepsilon$ and $\bar{y}=y / \sqrt{\varepsilon}$ near $(0,0)$ and using $\bar{x}$ and $\tilde{y}=(1-y) / \sqrt{\varepsilon}$ near $(1,1)$, we collect the terms of order $\varepsilon^{j}, 0 \leq j \leq n$. As a result, we find the equations for $\zeta_{l}^{j}\left(\right.$ or $\left.\zeta_{u}^{j}\right)$ :

$$
-\varepsilon \partial_{x}^{2} \zeta_{k}^{j}-\partial_{x} \zeta_{k}^{j}=\partial_{y}^{2} \zeta_{k}^{j-1} \text { in } \Omega, 0 \leq j \leq n, k=u \text { or } l .
$$


Here, we set $\zeta_{k}^{-1}=0$ for $k=l, u$.

At each order of $\varepsilon^{j}, 0 \leq j \leq n$, to cancel the error $\varphi^{j}$ near the corner $(0,0)$ or $(0,1)$, we supplement $(45)$ with the boundary conditions

$$
\begin{gathered}
\zeta_{k}^{j}=-\varphi_{k}^{j}(0, y) \quad \text { at } x=0,0 \leq j \leq n, k=l, u, \\
\zeta_{k}^{j} \longrightarrow 0 \quad \text { as } x \longrightarrow \infty, 0 \leq j \leq n, k=l, u .
\end{gathered}
$$

The explicit solutions $\zeta_{l}^{j}$ of (45) and (46), $0 \leq j \leq n$, are inductively found to be of the following form:

$$
\zeta_{l}^{j}=P^{j}\left(\frac{x}{\varepsilon}, \frac{y}{\sqrt{\varepsilon}}\right) \exp \left(\frac{-x}{\varepsilon}\right),
$$

where $P^{j}(x / \varepsilon, y / \sqrt{\varepsilon})$ is a polynomial in $x / \varepsilon$ of degree $j$ whose coefficients, independent of $\varepsilon$, are linear combinations of the quantities $\varepsilon^{s} \partial^{2 s} \varphi_{l}^{j-s}(0, y / \sqrt{\varepsilon}) / \partial y^{2 s}, 0 \leq s \leq j$. In the same manner, the explicit forms of the $\zeta_{u}^{j}, 0 \leq j \leq n$ can be found as well.

Using Lemma 1 and (47), we obtain the lemmas below.

Lemma 5. For each $0 \leq j \leq n$ and $k, s \geq 0$, one has the pointwise estimates:

$$
\begin{aligned}
\left|x^{k} y^{s} \partial_{x}^{i} \partial_{y}^{m} \zeta_{l}^{j}\right| \leq \kappa \varepsilon^{k-i+((s-m) / 2)} \exp \left(-c\left(\frac{x}{\varepsilon}+\frac{y}{\sqrt{\varepsilon}}\right)\right), \\
0 \leq i+m \leq 2 n+2-2 j, \\
\left|x^{k} y^{s} \partial_{x}^{i} \partial_{y}^{m} \zeta_{u}^{j}\right| \leq \kappa \varepsilon^{k-i+((s-m) / 2)} \exp \left(-c\left(\frac{x}{\varepsilon}+\frac{1-y}{\sqrt{\varepsilon}}\right)\right), \\
0 \leq i+m \leq 2 n+2-2 j,
\end{aligned}
$$

for a generic constant $c>0$ independent of $x, y$, and $\varepsilon$.

Lemma 6. For each $0 \leq j \leq n$ and $k, s \geq 0$, one has, for $0 \leq i+m \leq 2 n+2-2 j$,

$$
\begin{gathered}
\left\|x^{k} y^{s} \partial_{x}^{i} \partial_{y}^{m} \zeta_{l}^{j}\right\|_{L^{p}(\Omega)}+\left\|x^{k} y^{s} \partial_{x}^{i} \partial_{y}^{m} \zeta_{u}^{j}\right\|_{L^{p}(\Omega)} \\
\leq \kappa \varepsilon^{k-i+((s-m) / 2)+(3 / 2 p)}
\end{gathered}
$$

Thanks to (46) and Lemma 5, we estimate the values of $\zeta^{j}, 0 \leq j \leq n$, along the sides $x=1, y=0$, and $y=1$ :

$$
\zeta^{j}= \begin{cases}\text { e.s.t. } & \text { at } x=1, \\ \zeta_{l}^{j}(x, 0)+\text { e.s.t. } & \text { at } y=0, \\ \zeta_{u}^{j}(x, 0)+\text { e.s.t. } & \text { at } y=1 .\end{cases}
$$

Now from (43), (46), and (50), we observe that

$$
\begin{aligned}
& u^{\varepsilon}-\sum_{j=0}^{n} \varepsilon^{j}\left(u^{j}+\varphi^{j}+\xi^{j}+\theta^{j}+\zeta^{j}\right) \\
& = \begin{cases}0 & \text { at } x=0, \\
\sum_{j=0}^{n} \varepsilon^{j}\left(\theta^{j}+\zeta^{j}\right)=\text { e.s.t. } & \text { at } x=1, \\
\sum_{j=0}^{n} \varepsilon^{j}\left(\varphi_{u}^{j}+\theta^{j}+\zeta^{j}\right)=\sum_{j=0}^{n} \varepsilon^{j}\left(\theta^{j}+\zeta_{l}^{j}\right)+\text { e.s.t. } & \text { at } y=0, \\
\sum_{j=0}^{n} \varepsilon^{j}\left(\varphi_{l}^{j}+\theta^{j}+\zeta^{j}\right)=\sum_{j=0}^{n} \varepsilon^{j}\left(\theta^{j}+\zeta_{u}^{j}\right)+\text { e.s.t. } & \text { at } y=1 .\end{cases}
\end{aligned}
$$

3.5. Supplementary Correctors. Now, our task is to handle, at each order $0 \leq j \leq n$, the effects of $\theta^{j}$ and $\zeta^{j}$ along the top and bottom boundaries $y=0,1$. To this end, using (51), we construct the supplementary correctors $\eta^{j}$ in the form

$$
\eta^{j}=\eta_{l}^{j}+\eta_{u}^{j}, \quad 0 \leq j \leq n,
$$

where

$$
\begin{gathered}
\eta_{l}^{j}=-(1-y)\left(\theta^{j}+\zeta_{l}^{j}\right) \quad(x, y=0), \\
\eta_{u}^{j}=-y\left(\theta^{j}+\zeta_{u}^{j}\right) \quad(x, y=1) .
\end{gathered}
$$

From Lemmas 3 and 5, it is easy to verify the lemmas below.

Lemma 7. For each $0 \leq j \leq n$ and $s \geq 0$, one has the pointwise estimates:

$$
\left|x^{s} \partial_{x}^{i} \partial_{y}^{m} \eta^{j}\right| \leq \kappa \varepsilon^{s-i} \exp \left(-c \frac{x}{\varepsilon}\right), \quad 0 \leq i+m \leq 2 n+2-2 j,
$$

for a constant $c$ independent of $x, y$, and $\varepsilon$.

Lemma 8. For each $0 \leq j \leq n$ and $s \geq 0$, one has

$$
\left\|x^{s} \partial_{x}^{i} \partial_{y}^{m} \eta^{j}\right\|_{L^{p}(\Omega)} \leq \varepsilon^{s-i+(1 / p)}, \quad 0 \leq i+m \leq 2 n+2-2 j .
$$

Using (3), (14), (17), (22), (23), (38), and (46), we find that, near the corner $(0,0)$,

$$
\begin{aligned}
\left(\theta^{0}+\zeta_{l}^{0}\right)(0,0) & =g_{1}(0)-u^{0}(0,0)-\varphi_{l}^{0}(0,0) \\
& =g_{1}(0)-u^{0}(0,0)-\left(g_{3}(0)-u^{0}(0,0)\right)=0 .
\end{aligned}
$$

More generally, one can verify that

$$
\left(\theta^{j}+\zeta_{l}^{j}\right)(0,0)=0, \quad\left(\theta^{j}+\zeta_{u}^{j}\right)(0,1)=0, \quad 0 \leq j \leq n .
$$


Using (42), (50), and (57), we infer from (52) and (53) that, for $0 \leq j \leq n$,

$$
\eta^{j}= \begin{cases}0 & \text { at } x=0, \\ \text { e.s.t. } & \text { at } x=1, \\ -\left(\theta^{j}+\zeta_{l}^{j}\right) & \text { at } y=0, \\ -\left(\theta^{j}+\zeta_{u}^{j}\right) & \text { at } y=1 .\end{cases}
$$

Now, we finally obtain from (51) and (58) that

$$
\begin{aligned}
& u^{\varepsilon}-\sum_{j=0}^{n} \varepsilon^{j}\left(u^{j}+\varphi^{j}+\xi^{j}+\theta^{j}+\zeta^{j}+\eta^{j}\right) \\
& = \begin{cases}\sum_{j=0}^{n} \varepsilon^{j}\left(\theta^{j}+\zeta^{j}+\eta^{j}\right)=\text { e.s.t. } & \text { at } x=0, \\
\sum_{j=0}^{n} \varepsilon^{j}\left(\varphi_{u}^{j}+\theta^{j}+\zeta^{j}+\eta^{j}\right) & \text { at } x=1, \\
=\sum_{j=0}^{n} \varepsilon^{j}\left(\theta^{j}+\zeta_{l}^{j}+\eta^{j}\right)+\text { e.s.t. }=\text { e.s.t. } & \text { at } y=0, \\
\sum_{j=0}^{n} \varepsilon^{j}\left(\varphi_{l}^{j}+\theta^{j}+\zeta^{j}+\eta^{j}\right) & \\
=\sum_{j=0}^{n} \varepsilon^{j}\left(\theta^{j}+\zeta_{u}^{j}+\eta^{j}\right)+\text { e.s.t. }=\text { e.s.t. } & \text { at } y=1 .\end{cases}
\end{aligned}
$$

The e.s.t. on the right hand side of $(59)_{2,3,4}$ are caused, respectively, by $\left(\theta^{j}+\zeta^{j}+\eta^{j}\right)$ at $x=1,\left(\varphi_{u}^{j}+\zeta_{u}^{j}\right)$ at $y=0$, and $\left(\varphi_{l}^{j}+\zeta_{l}^{j}\right)$ at $y=1$.

\section{Asymptotic Error Analysis: The Main Result}

We define

$$
\begin{aligned}
\tilde{\rho} & :=\left.\left(u^{\varepsilon}-\sum_{j=0}^{n} \varepsilon^{j}\left(u^{j}+\Theta^{j}\right)\right)\right|_{\partial \Omega} \\
& =\text { (the right-hand side of (59)) }
\end{aligned}
$$

where $\Theta^{j}=\varphi^{j}+\xi^{j}+\theta^{j}+\zeta^{j}+\eta^{j}$ as defined in (11).

We construct an extension $\rho$ of $\tilde{\rho}$ in the form

$$
\begin{aligned}
\rho(x, y):= & r+\left.(1-x)(\tilde{\rho}-r)\right|_{x=0}+\left.x(\widetilde{\rho}-r)\right|_{x=1} \\
& +\left.(1-y)(\widetilde{\rho}-r)\right|_{y=0}+\left.y(\widetilde{\rho}-r)\right|_{y=1},
\end{aligned}
$$

where

$$
\begin{aligned}
r(x, y):= & \left.(1-x)(1-y) \tilde{\rho}\right|_{x=y=0}+\left.x(1-y) \tilde{\rho}\right|_{x=1, y=0} \\
& +\left.(1-x) y \widetilde{\rho}\right|_{x=0, y=1}+\left.x y \widetilde{\rho}\right|_{x=y=1} .
\end{aligned}
$$

Then one can verify that $\left.\rho\right|_{\partial \Omega}=\tilde{\rho}$ and

$$
\rho \text { is an e.s.t. in } \Omega \text {. }
$$

We set

$$
w_{\varepsilon n}:=u^{\varepsilon}-\sum_{j=0}^{n} \varepsilon^{j}\left(u^{j}+\Theta^{j}\right)-\rho .
$$

Then, using (1), (7), (11), (30), (34), (37), (45), (53), and (60), the equation of $w_{\varepsilon n}$ reads

$$
\begin{gathered}
L_{\varepsilon} w_{\varepsilon n}=\varepsilon^{n+1}\left(\Delta u^{n}+\partial_{x}^{2} \varphi^{n}+\partial_{y}^{2} \theta^{n}+\Xi_{1}^{n}\right) \\
+\varepsilon^{n}\left(\partial_{y}^{2} \theta^{n-1}+\varepsilon \partial_{y}^{2} \zeta^{n}+\Xi_{2}^{n}\right)+\text { e.s.t. } \text { in } \Omega, \\
w_{\varepsilon n}=0 \quad \text { on } \partial \Omega,
\end{gathered}
$$

where

$$
\begin{gathered}
\Xi_{1}=(1-y) \partial_{y}^{2} \theta^{n}(x, y=0)+y \partial_{y}^{2} \theta^{n} \quad(x, y=1), \\
\Xi_{2}=(1-y)\left(\partial_{y}^{2} \theta^{n-1}+\varepsilon \partial_{y}^{2} \zeta_{l}^{n}\right) \quad(x, y=0) \\
+y\left(\partial_{y}^{2} \theta^{n-1}+\varepsilon \partial_{y}^{2} \zeta_{u}^{n}\right) \quad(x, y=1) .
\end{gathered}
$$

We multiply (65) by $e^{x} w_{\varepsilon n}$ and integrate over $\Omega$. Then, using Lemmas 1, 3, and 5 and the Hardy inequality (see, e.g., $[2])$, we find that

$$
\begin{aligned}
& \varepsilon\left|w_{\varepsilon n}\right|_{H^{1}}^{2}+\frac{1-\varepsilon}{2}\left|w_{\varepsilon n}\right|_{L^{2}}^{2} \\
& \leq \kappa \varepsilon^{n+1}\left|\int_{\Omega}\left(\Delta u^{n}+\partial_{x}^{2} \varphi^{n}+\partial_{y}^{2} \theta^{n}+\Xi_{1}^{n}\right) w_{\varepsilon n} d x d y\right| \\
& \quad+\kappa \varepsilon^{n}\left|\int_{\Omega} x\left(\partial_{y}^{2} \theta^{n-1}+\varepsilon \partial_{y}^{2} \zeta^{n}+\Xi_{2}^{n}\right)\left(\frac{w_{\varepsilon n}}{x}\right) d x d y\right| \\
& \leq \kappa \varepsilon^{2 n+2}+\frac{1}{4}\left|w_{\varepsilon n}\right|_{L^{2}}^{2}+\frac{\varepsilon}{2}\left|w_{\varepsilon n}\right|_{H^{1}}^{2} .
\end{aligned}
$$

Thanks to the elliptic regularity theory and (67), we also find that

$$
\begin{aligned}
\left\|w_{\varepsilon n}\right\|_{H^{2}(\Omega)} & \leq \kappa\left\|\Delta w_{\varepsilon n}\right\|_{L^{2}(\Omega)} \\
& \leq \kappa \varepsilon^{-1} \| \partial_{x} w_{\varepsilon n}+\text { lower order terms } \|_{L^{2}(\Omega)} \\
& \leq \kappa \varepsilon^{n-(1 / 2)} .
\end{aligned}
$$

Finally, from (63), (64), (67), and (68), we obtain the convergence result on the difference of $u^{\varepsilon}$ and the proposed asymptotic expansions that we summarize in Theorem 9.

Theorem 9. For each fixed $n \geq 0$, as the diffusivity parameter $\varepsilon$ vanishes, the difference between the solution $u^{\varepsilon}$ of (1) and its asymptotic expansion (6) converges to zero in the following sense:

$$
\begin{gathered}
\left\|u^{\varepsilon}-\sum_{j=0}^{n} \varepsilon^{j}\left(u^{j}+\Theta^{j}\right)\right\|_{\varepsilon} \leq \kappa \varepsilon^{n+1}, \\
\left\|u^{\varepsilon}-\sum_{j=0}^{n} \varepsilon^{j}\left(u^{j}+\Theta^{j}\right)\right\|_{H^{2}(\Omega)} \leq \kappa \varepsilon^{n-(1 / 2)} .
\end{gathered}
$$


Remark 10. Due to the continuity of $g$ as appearing in (3), $\xi^{0}$ can be omitted in (11) for $j=0$ at order $\varepsilon^{0}$. Then one can verify that

$$
\left\|u^{\varepsilon}-\left(u^{0}+\varphi^{0}+\theta^{0}+\zeta^{0}+\eta^{0}\right)\right\|_{\varepsilon} \leq \kappa \varepsilon^{3 / 4}
$$

which gives a convergence estimate that a factor of $\varepsilon^{1 / 4}$ is worse than what is stated in (69) for $n=0$.

\section{Appendix}

\section{A. Proof of Lemma 1}

To prove (27), for $\bar{y}=y / \sqrt{\varepsilon}$, since $y^{s} \exp (-c \bar{y}) \leq$ $\kappa \varepsilon^{s / 2} \exp \left(-c_{0} \bar{y}\right)$, for all $y, 0<c_{0}<c$, it suffices to prove that

$$
\begin{gathered}
\left|\partial_{x}^{i} \partial_{\bar{y}}^{m} \varphi_{l}^{j}\right| \leq \kappa \exp \left(-c \frac{\bar{y}}{\sqrt{1-x}}\right), \\
0 \leq i+m \leq 2 n+2-2 j, \quad i, m \geq 0 .
\end{gathered}
$$

Note that (28) would follow similarly. Since $h^{j}(x)=h_{0}^{j}(x)=$ $-u^{j}(x, i)-\gamma_{0}^{j}(x)$ for $j \geq 1$ and $g_{3}(x)-u^{0}(x, 0)-\gamma_{0}^{0}(x)$ for $j=0$; thanks to (3), we note that $\partial_{x}^{k} h^{j}(1)=0$ for $0 \leq k \leq$ $2 n+1-2 j$.

Now, to prove (A.1), we use an induction on $j$.

(1) We begin with $j=0$.

From (25), since $\partial_{x}^{k} h^{0}(1)=0$ for $0 \leq k \leq 2 n+1$, differentiating $\varphi_{l}^{0}$ in $x$ we find that

$$
\begin{aligned}
\left|\partial_{x}^{i} \varphi_{l}^{0}\right| & =\sqrt{\frac{2}{\pi}}\left|\int_{\bar{y} / \sqrt{2(1-x)}}^{\infty} \exp \left(-\frac{y_{1}^{2}}{2}\right) \partial_{x}^{i} h^{0}\left(x+\frac{\bar{y}^{2}}{2 y_{1}^{2}}\right) d y_{1}\right| \\
& \leq \kappa \int_{\bar{y} / \sqrt{2(1-x)}}^{\infty} \exp \left(-\frac{y_{1}^{2}}{2}\right) d y_{1}, \quad 0 \leq i \leq 2 n+2 .
\end{aligned}
$$

Using $\exp \left(-y_{1}^{2} / 2\right) \leq \kappa \exp \left(-c y_{1}\right)$, for all $y_{1} \geq 0$, for some $c>0$, (A.1) for $m=0$ is proved. Since $-\varphi_{l \bar{y} \bar{y}}^{0}-\varphi_{l x}^{0}=0$, for $m=2(k+1), k \geq 0$, from (A.2) we easily find that

$$
\begin{aligned}
\left|\partial_{x}^{i} \partial_{\bar{y}}^{2(k+1)} \varphi_{l}^{0}\right|=\left|\partial_{x}^{i+k+1} \varphi_{l}^{0}\right| & \leq \kappa \exp \left(-c \frac{\bar{y}}{\sqrt{1-x}}\right), \\
0 & \leq i+k+1 \leq 2 n+2 ;
\end{aligned}
$$

thus (A.3) holds for $0 \leq i+m=i+2(k+1) \leq k+1+2 n+2$, and this implies that (A.1) holds for $m=2(k+1), k \geq 0, j=0$.
Since $\partial_{x}^{i+1} h^{0}(1)=0,0 \leq i+1 \leq 2 n+1$, we now notice that

$$
\begin{aligned}
&\left|\partial_{x}^{i} \partial_{\bar{y}} \varphi_{l}^{0}\right|= \sqrt{\frac{2}{\pi}} \mid \int_{\bar{y} / \sqrt{2(1-x)}}^{\infty} \exp \left(-\frac{y_{1}^{2}}{2}\right) \partial_{x}^{i+1} h^{0} \\
& \times\left(x+\frac{\bar{y}^{2}}{2 y_{1}^{2}}\right) \frac{\bar{y}}{y_{1}^{2}} d y_{1} \mid \\
& \leq \kappa \bar{y} \int_{\bar{y} / \sqrt{2(1-x)}}^{\infty} \exp \left(-\frac{y_{1}^{2}}{2}\right) d\left(-y_{1}^{-1}\right), \\
& 0 \leq i+1 \leq 2 n+2 .
\end{aligned}
$$

Integrating by parts in the last integral, we deduce that

$$
\begin{aligned}
& \left|\partial_{x}^{i} \partial_{\bar{y}} \varphi_{l}^{0}\right| \\
& \leq \kappa\left(\sqrt{1-x} \exp \left(-\frac{\bar{y}^{2}}{4(1-x)}\right)\right. \\
& \left.+\bar{y} \int_{\bar{y} / \sqrt{2(1-x)}}^{\infty} \exp \left(-\frac{y_{1}^{2}}{2}\right) d y_{1}\right) \\
& \leq \kappa\left(\exp \left(-c \frac{\bar{y}}{\sqrt{1-x}}\right)+\bar{y} \exp \left(-c \frac{\bar{y}}{\sqrt{1-x}}\right)\right) \\
& \leq\left(\text { since } t \exp (-c t) \leq \kappa \exp \left(-c_{0} t\right)\right. \text {, } \\
& \forall t \geq 0 \text {, for some } 0<c_{0}<c \text { ) } \\
& \leq \kappa \exp \left(-c_{0} \frac{\bar{y}}{\sqrt{1-x}}\right), \quad 0 \leq i+1 \leq 2 n+2 .
\end{aligned}
$$

Hence, for $m=2 k+1, k \geq 0$, using $-\varphi_{l \overline{y y}}^{0}-\varphi_{l x}^{0}=0$ again, we note from (A.5) that

$$
\begin{aligned}
\left|\partial_{x}^{i} \partial_{\bar{y}}^{2 k+1} \varphi_{l}^{0}\right|=\left|\partial_{x}^{i+k} \partial_{\bar{y}} \varphi_{l}^{0}\right| \leq & \kappa \exp \left(-c \frac{\bar{y}}{\sqrt{1-x}}\right), \\
0 & \leq i+k+1 \leq 2 n+2 ;
\end{aligned}
$$

thus (A.6) holds for $0 \leq i+m=i+2 k+1 \leq k+2 n+2$, and this proves (A.1) for $m=2 k+1, k \geq 0, j=0$. Hence, (A.1) is proved for $j=0$.

(2) We now assume that (A.1) holds at any order less than or equal to $j-1$, and we want to prove it at the order $j$.

At order $j \geq 1$, we perform the analysis as follows.

We first note that the homogeneous solution of $\varphi_{l}^{j}$, that is the first integral of (26) for $\varphi_{l}^{j}$, can be similarly estimated. We just replace $h_{0}^{0}(x)$ by $h_{0}^{j}(x)$ in the above analysis.

Since $\partial_{x}^{k} h^{j}(1)=0$ for $0 \leq k \leq 2 n+1-2 j$, the first integral is then estimated as in (A.1) for $j=0$. We just replace the above $2 n+2$ by $2 n+2-2 j$. We now estimate the particular solution of $\varphi_{l}^{j}$, that is, the second integral of (26) denoted by $I$. For simplicity in the analysis below, we write that

$$
J=\exp \left(-\frac{\left(\bar{y}-y_{1}\right)^{2}}{4 x_{1}}\right)-\exp \left(-\frac{\left(\bar{y}+y_{1}\right)^{2}}{4 x_{1}}\right) .
$$


Using the induction assumption at order $j-1$, from (A.1) for $j-1$, we note that for $0 \leq 2+i \leq 2 n+2-2(j-1)$, that is, $0 \leq i \leq 2 n+2-2 j$,

$$
\begin{gathered}
\partial_{x}^{2+i} \varphi_{l}^{j-1}\left(1^{-}, y_{1}\right)=0 \\
\left|\partial_{x}^{2+i} \varphi_{l}^{j-1}\left(x+x_{1}, y_{1}\right)\right| \leq \kappa \exp \left(-c \frac{y_{1}}{\sqrt{1-x-x_{1}}}\right) \\
\leq \kappa \exp \left(-c \frac{y_{1}}{\sqrt{1-x}}\right) .
\end{gathered}
$$

Thanks to (A.8), differentiating $I$, the second integral of (26), we find that

$$
\begin{array}{r}
\partial_{x}^{i} I=\frac{1}{2 \sqrt{\pi}} \int_{0}^{1-x} \int_{0}^{\infty} \frac{J}{\sqrt{x_{1}}} \partial_{x}^{2+i} \varphi_{l}^{j-1}\left(x+x_{1}, y_{1}\right) d y_{1} d x_{1}, \\
0 \leq i \leq 2 n+2-2 j .
\end{array}
$$

Since $0 \leq x_{1} \leq 1-x \leq 1,|J| \leq 2 \exp \left(-\left(\bar{y}-y_{1}\right)^{2} / 4 x_{1}\right) \leq$ $2 \exp \left(-\left(\bar{y}-y_{1}\right)^{2} / 4(1-x)\right)$, from (A.9) we find that for $0 \leq i \leq$ $2 n+2-2 j$,

$$
\begin{aligned}
\left|\partial_{x}^{i} I\right| \leq & \kappa \int_{0}^{1-x} \frac{1}{\sqrt{x_{1}}} d x_{1} \\
& \times \int_{0}^{\infty} \exp \left(-\frac{\left(\bar{y}-y_{1}\right)^{2}}{4(1-x)}\right) \exp \left(-c \frac{y_{1}}{\sqrt{1-x}}\right) d y_{1} \\
\leq & (\operatorname{setting} \beta=2 c \sqrt{1-x}) \\
\leq & \kappa \int_{0}^{\infty} \exp \left(-\frac{\left(y_{1}-\bar{y}+\beta\right)^{2}}{4(1-x)}\right. \\
\leq & \kappa \exp \left(-c \frac{\beta}{\sqrt{1-x}}\right) \int_{0}^{\infty} \exp \left(-\frac{\left(y_{1}-\bar{y}+\beta\right)^{2}}{4(1-x)}\right) d y_{1} \\
\leq & \kappa \exp \left(-c \frac{\bar{y}}{\sqrt{1-x}}\right) \cdot
\end{aligned}
$$

This proves (A.1) for $m=0, j \geq 1$.

For $m=2(k+1), k \geq 0$, thanks to the induction assumption on $j-1$, from (A.1) we note that for $0 \leq i+s+$ $2+2(k-s) \leq 2 n+2-2(j-1)$,

$$
\left|\partial_{x}^{i+s+2} \partial_{\bar{y}}^{2(k-s)} \varphi_{l}^{j-1}\right| \leq \kappa \exp \left(-c \frac{\bar{y}}{\sqrt{1-x}}\right),
$$

from (A.11) we also note that

$$
\begin{aligned}
& \left|\partial_{x}^{i+k+1} I\right| \leq \kappa \exp \left(-c \frac{\bar{y}}{\sqrt{1-x}}\right) \\
& \text { for } 0 \leq i+k+1 \leq 2 n+2-2 j .
\end{aligned}
$$

Since $-I_{\overline{y y}}-I_{x}=\varphi_{l x x}^{j-1}$, we thus find that

$$
\begin{aligned}
\left|\partial_{x}^{i} \partial_{\bar{y}}^{2(k+1)} I\right| & \leq\left|\partial_{x}^{i+k+1} I\right|+\sum_{s=0}^{k}\left|\partial_{x}^{i+s+2} \partial_{\bar{y}}^{2(k-s)} \varphi_{l}^{j-1}\right| \\
& \leq \kappa \exp \left(-c \frac{\bar{y}}{\sqrt{1-x}}\right),
\end{aligned}
$$

for $0 \leq i+2(k+1) \leq 2 n+2-2 j$.

This proves (A.1) for $m=2(k+1), k \geq 0, j \geq 1$.

Since $\partial_{\bar{y}} \exp \left(-\left(\bar{y}-y_{1}\right)^{2} / 4 x_{1}\right)=-\partial_{y_{1}} \exp \left(-\left(\bar{y}-y_{1}\right)^{2} / 4 x_{1}\right)$, differentiating (A.10) in $\bar{y}$ we observe that

$\left|\partial_{x}^{i} \partial_{\bar{y}} I\right|$

$$
\begin{aligned}
& \leq \kappa\left|\int_{0}^{1-x} \frac{1}{\sqrt{x_{1}}} \int_{0}^{\infty} \partial_{\bar{y}} J \cdot \partial_{x}^{2+i} \varphi_{l}^{j-1}\left(x+x_{1}, y_{1}\right) d y_{1} d x_{1}\right| \\
& =\kappa\left|\int_{0}^{1-x} \frac{1}{\sqrt{x_{1}}} \int_{0}^{\infty} \partial_{y_{1}} \tilde{J} \cdot \partial_{x}^{2+i} \varphi_{l}^{j-1}\left(x+x_{1}, y_{1}\right) d y_{1} d x_{1}\right|
\end{aligned}
$$

$\leq$ (integrating by parts in $\left.y_{1}\right)$

$$
\begin{aligned}
& \leq \kappa\left|\int_{0}^{1-x} \frac{1}{\sqrt{x_{1}}} \exp \left(-\frac{\bar{y}^{2}}{4 x_{1}}\right) \partial_{x}^{2+i} \varphi_{l}^{j-1}\left(x+x_{1}, 0\right) d x_{1}\right| \\
& \quad+\kappa\left|\int_{0}^{1-x} \frac{1}{\sqrt{x_{1}}} \int_{0}^{\infty} \tilde{J} \cdot \partial_{x}^{2+i} \partial_{y_{1}} \varphi_{l}^{j-1}\left(x+x_{1}, y_{1}\right) d y_{1} d x_{1}\right|
\end{aligned}
$$

where $\tilde{J}=\exp \left(-\left(\bar{y}-y_{1}\right)^{2} / 4 x_{1}\right)+\exp \left(-\left(\bar{y}+y_{1}\right)^{2} / 4 x_{1}\right) \leq$ $2 \exp \left(-\left(\bar{y}-y_{1}\right)^{2} / 4(1-x)\right)$. Here, we note that $\partial_{\bar{y}} J=-\partial_{y_{1}} J$. Thanks to the induction assumption on $j-1$, from (A.1) we thus note that for $0 \leq 2+i+1 \leq 2 n+2-2(j-1)$, that is, $0 \leq i \leq 2 n+1-2 j$,

$$
\left|\partial_{x}^{2+i} \partial_{y_{1}} \varphi_{l}^{j-1}\left(x+x_{1}, y_{1}\right)\right| \leq \kappa \exp \left(-c \frac{y_{1}}{\sqrt{1-x}}\right),
$$

and for $0 \leq 2+i \leq 2 n+2-2(j-1)$, that is, $0 \leq i \leq 2 n+2-2 j$,

$$
\left|\partial_{x}^{2+i} \partial_{y_{1}} \varphi_{l}^{j-1}\left(x+x_{1}, 0\right)\right| \leq \kappa .
$$

As in (A.11) we similarly find that for $0 \leq i \leq 2 n+1-2 j$,

$$
\left|\partial_{x}^{i} \partial_{\bar{y}} I\right| \leq \kappa \exp \left(-c \frac{\bar{y}}{\sqrt{1-x}}\right) \text {. }
$$

Hence, for $m=2 k+1, k \geq 0$, using the induction assumption at order $j-1$, from (A.1) we note that for $0 \leq i+s+2+2(k-$ s) $-1 \leq 2 n+2-2(j-1)$,

$$
\left|\partial_{x}^{i+s+2} \partial_{\bar{y}}^{2(k-s)-1} \varphi_{l}^{j-1}\right| \leq \kappa \exp \left(-c \frac{\bar{y}}{\sqrt{1-x}}\right),
$$

from (A.18) we also note that

$$
\begin{array}{r}
\left|\partial_{x}^{i+k} \partial_{\bar{y}} I\right| \leq \kappa \exp \left(-c \frac{\bar{y}}{\sqrt{1-x}}\right), \\
\text { for } 0 \leq i+k \leq 2 n+1-2 j .
\end{array}
$$


Using $-I_{\overline{y y}}-I_{x}=\varphi_{l x x}^{j-1}$ again we thus find that

$$
\begin{aligned}
& \left|\partial_{x}^{i} \partial_{\bar{y}}^{2 k+1} I\right| \\
& \quad \leq\left|\partial_{x}^{i+k} \partial_{\bar{y}} I\right|+\sum_{s=0}^{k-1}\left|\partial_{x}^{i+s+2} \partial_{\bar{y}}^{2(k-s)-1} \varphi_{l}^{j-1}\right| \\
& \quad \leq \kappa \exp \left(-c \frac{\bar{y}}{\sqrt{1-x}}\right), \text { for } 0 \leq i+2 k+1 \leq 2 n+2-2 j .
\end{aligned}
$$

This proves (A.1) for $m=2 k+1, k \geq 0, j \geq 1$. Hence, the lemma is proved.

\section{B. Study of the Elliptic Corner$$
\text { Correctors } \xi^{j}=\xi_{l}^{j}+\xi_{u}^{j}
$$

For each $0 \leq j \leq n$, using the stretched variables

$$
X=\frac{1-x}{2 \varepsilon}, \quad Y=\frac{y}{2 \varepsilon},
$$

one can define an approximation $\bar{\xi}_{l}^{j}$ of $\xi_{l}^{j}$ as a solution of the system,

$$
\begin{gathered}
-\partial_{X}^{2} \bar{\xi}_{l}^{j}-\partial_{Y}^{2} \bar{\xi}_{l}^{j}+2 \partial_{X} \bar{\xi}_{l}^{j}=0, \\
\bar{\xi}_{l}^{j}=0 \quad \text { at } X=0, \\
\bar{\xi}_{l}^{j}=\gamma_{0}^{j}(1-2 \varepsilon X) \quad \text { at } Y=0, \\
\bar{\xi}_{l}^{j} \longrightarrow 0 \quad \text { at } X^{2}+Y^{2} \longrightarrow \infty, Y>0 .
\end{gathered}
$$

The explicit expression of $\bar{\xi}^{j}$ is given as (see [1])

$$
\begin{aligned}
\bar{\xi}_{l}^{j}=\frac{Y}{\pi} \int_{0}^{\infty} & {\left[\frac{K_{1}\left(s_{1}\right)}{s_{1}}-\frac{K_{1}\left(s_{2}\right)}{s_{2}}\right] } \\
& \times \gamma_{0}^{j}(1-2 \varepsilon s) \exp (-(s-X)) d s
\end{aligned}
$$

where $K_{1}$ is the modified Bessel function of the second kind of the first order, and

$$
s_{1}=\sqrt{(X-s)^{2}+Y^{2}}, \quad s_{2}=\sqrt{(X+s)^{2}+Y^{2}} .
$$

Using an appropriate barrier function and the maximum principle (see [1]), one can verify that

$$
\left|\bar{\xi}_{l}^{j}(X, Y)\right| \leq \kappa \exp \left(-c\left(\sqrt{X^{2}+Y^{2}}-X\right)\right)
$$

for a constant $c$ independent of $\varepsilon$. An approximation $\bar{\xi}_{u}^{j}$ of $\xi_{u}^{j}$ can be constructed in the same manner.

Using (33) and (B.5), we notice that $\xi^{j}-\left(\bar{\xi}_{l}^{j}+\bar{\xi}_{u}^{j}\right)=$ e.s.t. at $y=0,1$. We deduce from (17), (18), and (B.2) 2 that $\gamma_{k}^{j}(1)=$ 0 , and hence $\xi^{j}-\left(\bar{\xi}_{l}^{j}+\bar{\xi}_{u}^{j}\right)=0$ at $x=1$. Moreover, using
Lemma B.1 below, we see that $\xi^{j}-\left(\bar{\xi}_{l}^{j}+\bar{\xi}_{u}^{j}\right)=0$ at $x=0$. Then, using these observations, thanks to the maximum principle, we find that

$$
\left|\xi^{j}-\left(\bar{\xi}_{l}^{j}+\bar{\xi}_{u}^{j}\right)\right|=\text { e.s.t., } \quad 0 \leq j \leq n,
$$

which implies that the elliptic corrector $\xi^{j}, 0 \leq j \leq n$, satisfies the pointwise estimates similar to (B.5) as for its approximation $\bar{\xi}^{j}=\bar{\xi}_{l}^{j}+\bar{\xi}_{u}^{j}$.

Lemma B.1. There exists a positive constant $\kappa$, independent of $\varepsilon$, such that

$$
\left|\bar{\xi}^{j}(X, Y)\right| \leq \kappa \min \{U(X), U(Y)\}, \quad 0 \leq j \leq n,
$$

where

$$
U(Z)= \begin{cases}1 & \text { if } 0 \leq Z \leq a, \\ 1-4(Z-a)^{2} & \text { if } a<Z \leq b, \\ 0 & \text { if } Z>b,\end{cases}
$$

and $a=3 / 8 \varepsilon, b=a+(1 / 2)$.

To prove Lemma B.1, we will recall below the weak maximum principle (see, e.g., [17]).

We consider an operator $L$ of the form,

$$
L u=\sum_{i, j=1}^{m} D_{i}\left(a^{i j} D_{j} u\right)+\sum_{i=1}^{m}\left(D_{i}\left(b^{i} u\right)+c^{i} D_{i} u\right)+d u
$$

where the coefficients $a^{i j}, b^{i}, c^{i}$, and $d$ are assumed to be measurable functions in a domain $\Omega_{m} \subset \mathbb{R}^{m}$, and $D_{i}$ denotes $\partial / \partial x_{i}, 1 \leq i \leq m$.

We assume that $L$ is strictly elliptic in $\Omega_{m}$, that is, there exists $\lambda>0$, such that

$$
\sum_{i, j=1}^{m} a^{i j} \xi_{i} \xi_{j} \geq \lambda|\xi|^{2}, \quad \forall \xi \in \mathbb{R}^{m}
$$

and that $L$ has bounded coefficients, that is, for some constants $\Lambda$ and $\nu \geq 0$, we have

$$
\sum_{i, j=1}^{m}\left|a^{i j}\right|^{2} \leq \Lambda^{2}, \quad \lambda^{-2} \sum_{i=1}^{m}\left(\left|b^{i}\right|^{2}+\left|c^{i}\right|^{2}\right)+\lambda^{-1}|d| \leq v^{2} .
$$

We also assume that

$$
\int_{\Omega_{m}}\left(d v-\sum_{i=1}^{m} b^{i} D_{i} v\right) \leq 0, \quad \forall v \geq 0, v \in \mathscr{C}_{c}^{1}\left(\Omega_{m}\right) .
$$

We say that a weakly differentiable function $u$ satisfies $L u=0,(\geq 0$ or $\leq 0)$, in the weak sense, if

$$
\int_{\Omega_{m}}\left(\sum_{i, j=1}^{m} a^{i j} D_{j} u D_{i} v+\sum_{i=1}^{m}\left(b^{i} u D_{i} v-c^{i} D_{i} u v\right)-d u v\right)=0
$$

$$
(\leq 0 \text { or } \geq 0) \text {, }
$$


for all nonnegative functions $v \in \mathscr{C}_{c}^{1}\left(\Omega_{m}\right)$, provided that $a^{i j} D_{j} u+b^{i} u$ and $c^{i} D_{i} u+d u, 1 \leq i \leq m$, are locally integrable.

Now we state the following maximum principle.

Lemma B.2. Under the conditions (B.10), (B.11), and (B.12), if $u \in H^{1}\left(\Omega_{m}\right)$ satisfies $L u \geq 0$, or $L u \leq 0$, in the weak sense, then one has, respectively,

$$
\sup _{\Omega_{m}} u \leq \sup _{\partial \Omega_{m}} u^{+} \text {or } \quad \inf _{\Omega_{m}} u \geq \inf _{\partial \Omega_{m}} u^{-} .
$$

Thanks to Lemma B.2, we can now prove Lemma B.1.

Proof of Lemma B.1. Given $\varepsilon_{0}>0$, thanks to (B.2) ${ }_{4}$, we may choose a region $\Omega_{0}=\left\{(X, Y) \in[0, \infty) \times[0, \infty) \mid X^{2}+Y^{2} \leq\right.$ $\left.R^{2}\right\}$ with $R$ sufficiently large so that $\left|\bar{\xi}^{j}\right|<\varepsilon_{0}$ on the circle $X^{2}+Y^{2}=R^{2}, X, Y>0$. We then define the barrier function

$$
\widetilde{U}=\widetilde{U}(X, Y)=C U(X)+\varepsilon_{0}, \quad(X, Y) \in[0, \infty) \times[0, \infty),
$$

where $U(X)$ is given in (B.8). Here, $C$ is a positive constant and it will be determined below. Note that $\widetilde{U}(X, Y) \in H^{1}\left(\Omega_{0}\right)$.

Going back to the elliptic problem (B.2) and writing the elliptic operator $L$ as

$$
L \bar{\xi}^{j}:=\bar{\xi}_{X X}^{j}+\bar{\xi}_{Y Y}^{j}-2 \bar{\xi}_{X}^{j},
$$

we find that the operator $L$ satisfies (B.10), (B.11), and (B.12). We also find that for $v \in \mathscr{C}_{c}^{1}\left(\Omega_{0}\right), v \geq 0$,

$$
\begin{aligned}
\int_{\Omega_{0}}\left(\widetilde{U}_{X} v_{X}+\widetilde{U}_{Y} v_{Y}+2 \widetilde{U}_{X} v\right) \\
\quad=\iint_{0}^{R} C\left(U_{X} v_{X}+2 U_{X} v\right) d X d Y \geq 0 .
\end{aligned}
$$

Indeed, if $0 \leq X \leq a$ or $X>b\left(a, b\right.$ as in (B.8)), since $U_{X}=0$, we only have to consider the case $a<R \leq b$. For this $R$, since $U \in H^{2}\left(\Omega_{0}\right)$, we note that $\int_{0}^{R}\left(U_{X} v_{X}+2 U_{X} v\right) d X=\int_{0}^{R}\left(-U_{X X}+\right.$ $\left.2 U_{X}\right) v d X=\int_{a}^{R}\left(-U_{X X}+2 U_{X}\right) v d X \geq 0$ for all non-negative $v \in \mathscr{C}_{c}^{1}\left(\Omega_{0}\right)$.

Since $L\left( \pm \bar{\xi}^{j}\right)=0$, we thus find that $L\left(\widetilde{U} \pm \bar{\xi}^{j}\right) \leq 0$ in the weak sense.

Thanks to the cut-off function $\delta(x)$, choosing $C=$ $\max _{x \in[1 / 2,1]}\left|\widetilde{\gamma}^{j}(x)\right|$, we find that

$$
\begin{aligned}
& \widetilde{U}(X, 0)=C U(X)+\varepsilon_{0} \geq\left|\tilde{\gamma}^{j}(x)\right| \chi_{[1 / 2,1]}(x) \\
&=\left|\tilde{\gamma}^{j}(1-2 \varepsilon X)\right| \chi_{[0,1 / 4 \varepsilon]}(X) \geq\left|P^{j}(X)\right| \\
&=\left|\bar{\xi}^{j}(X, 0)\right|, \\
& \widetilde{U}(0, Y)=C+\varepsilon_{0} \geq 0=\left|\bar{\xi}^{j}(0, Y)\right|, \\
& \widetilde{U}(X, Y) \geq \varepsilon_{0}>\left|\bar{\xi}^{j}(X, Y)\right|
\end{aligned}
$$

on $\partial \Omega_{0}$ with $X^{2}+Y^{2}=R^{2}, X, Y>0$.
Thus, we note that $\widetilde{U} \pm \bar{\xi}^{j} \geq 0$ on $\partial \Omega_{0}$. Using Lemma B.2 we conclude that, for $(X, Y) \in \Omega_{0}$,

$$
\left(\widetilde{U} \pm \bar{\xi}^{j}\right)(X, Y) \geq \inf _{\Omega_{0}}\left(\widetilde{U} \pm \bar{\xi}^{j}\right) \geq \inf _{\partial \Omega_{0}}\left(\widetilde{U} \pm \bar{\xi}^{j}\right)^{-}=0 .
$$

Hence, we have

$$
\left|\bar{\xi}^{j}(X, Y)\right| \leq \widetilde{U}(X, Y)=C U(X)+\varepsilon_{0} .
$$

Letting $R \rightarrow \infty$, we deduce that $\left|\bar{\xi}^{j}(X, Y)\right| \leq C U(X)$. Similarly, we can conclude that $\left|\bar{\xi}^{j}(X, Y)\right| \leq C U(Y)$. This proves the lemma.

\section{Acknowledgments}

This work was supported in part by the Research Fund of Indiana University, by NSF Grants DMS 0906440, DMS 1206438, and DMS 1212141, and by Basic Science Research Program through the National Research Foundation of Korea (NRF) funded by the Ministry of Education, Science and Technology (2012001167).

\section{References}

[1] S.-D. Shih and R. B. Kellogg, "Asymptotic analysis of a singular perturbation problem," SIAM Journal on Mathematical Analysis, vol. 18, no. 5, pp. 1467-1511, 1987.

[2] G. H. Hardy, J. E. Littlewood, and G. Pólya, Inequalities, Cambridge University Press, Cambridge, UK, 1988.

[3] L. Prandtl, "Verber Flüssigkeiten bei sehr kleiner Reibung," in Proceedings of the Verk. III Intem. Math. Kongr. Heidelberg, pp. 484-491, Teuber, Leibzig, Germany, 1905.

[4] L. Prandtl, "Über Flüssigkeitsbewegung bei sehr kleiner Reibung," in Verhandlung des 3 Internat. Math. Kongr. Heidelberg, pp. 484-491, Teubner, Leipzig, Germany, 1905.

[5] L. Prandtl, Gesammelte Abhandlungen zur angewandten Mechanik, Hydro- und Aerodynamik, edited by W. Tollmien, $\mathrm{H}$. Schlichting, and H. Görtler, Springer, Berlin, Germany, 1961.

[6] L. Prandtl, "Über Flüssigkeitsbewegung bei sehr kleiner Reibung," in Gesammelte Abhandlungen zur angewandten Mechanik, Hydro- und Aerodynamik, W. Tollmien, H. Schlichting, and H. Görtler, Eds., Springer, Berlin, Germany, 1961.

[7] J. B. Rauch and F. J. Massey, III, "Differentiability of solutions to hyperbolic initial-boundary value problems," Transactions of the American Mathematical Society, vol. 189, pp. 303-318, 1974.

[8] S. Smale, "Smooth solutions of the heat and wave equations," Commentarii Mathematici Helvetici, vol. 55, no. 1, pp. 1-12, 1980.

[9] R. Temam, "Behaviour at time $t=0$ of the solutions of semilinear evolution equations," Journal of Differential Equations, vol. 43, no. 1, pp. 73-92, 1982.

[10] Q. Chen, Z. Qin, and R. Temam, "Numerical resolution near $t=0$ of nonlinear evolution equations in the presence of corner singularities in space dimension 1," Communications in Computational Physics, vol. 9, no. 3, pp. 568-586, 2011.

[11] N. Flyer and B. Fornberg, "Accurate numerical resolution of transients in initial-boundary value problems for the heat equation," Journal of Computational Physics, vol. 184, no. 2, pp. 526-539, 2003 
[12] N. Flyer and B. Fornberg, "On the nature of initial-boundary value solutions for dispersive equations," SIAM Journal on Applied Mathematics, vol. 64, no. 2, pp. 546-564, 2004.

[13] G.-M. Gie, "Asymptotic expansion of the Stokes solutions at small viscosity: the case of non-compatible initial data," Communications in Mathematical Sciences, to appear.

[14] J. R. Cannon, The One-Dimensional Heat Equation, vol. 23 of Encyclopedia of Mathematics and Its Applications, AddisonWesley Publishing Company Advanced Book Program, Reading, Mass, USA, 1984.

[15] C.-Y. Jung and R. Temam, "Numerical approximation of two-dimensional convection-diffusion equations with multiple boundary layers," International Journal of Numerical Analysis and Modeling, vol. 2, no. 4, pp. 367-408, 2005.

[16] G.-M. Gie, M. Hamouda, and R. Temam, "Boundary layers in smooth curvilinear domains: parabolic problems," Discrete and Continuous Dynamical Systems Series A, vol. 26, no. 4, pp. 12131240, 2010.

[17] D. Gilbarg and N. S. Trudinger, Elliptic Partial Differential Equations of Second Order, vol. 224 of Fundamental Principles of Mathematical Sciences, Springer, Berlin, Germany, 2nd edition, 1983. 


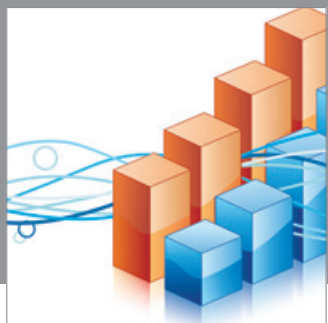

Advances in

Operations Research

mansans

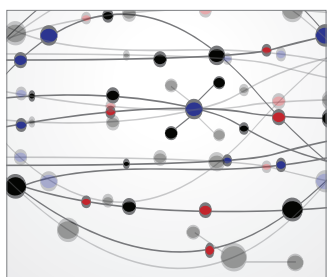

The Scientific World Journal
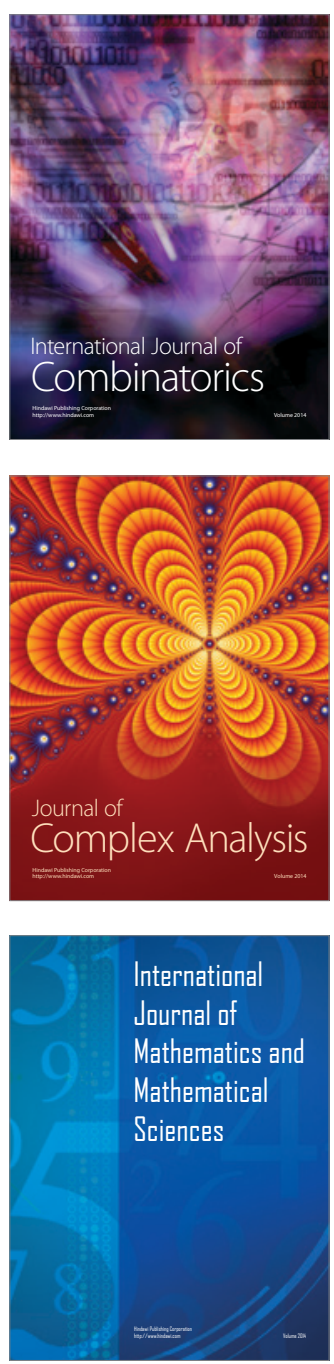
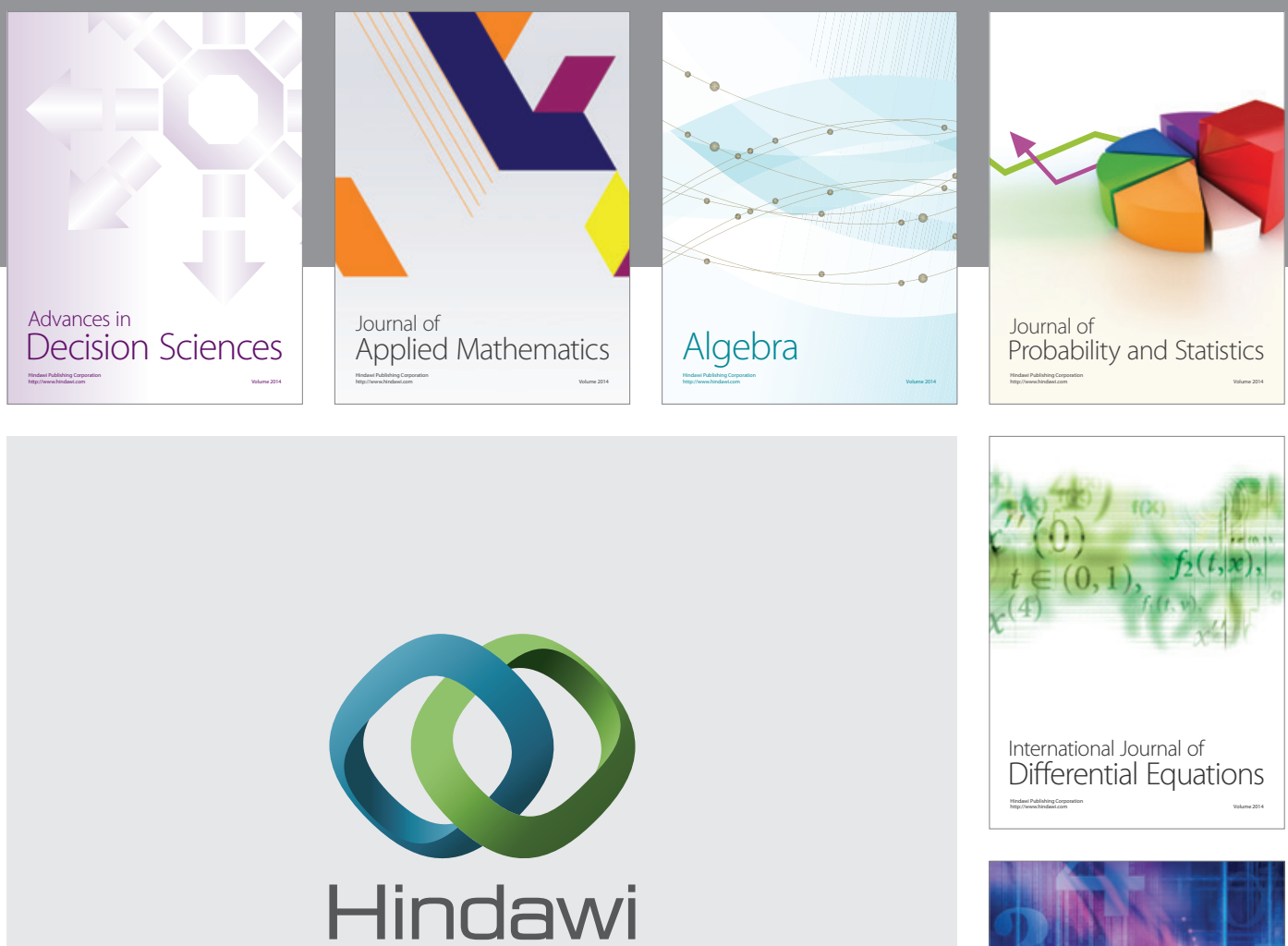

Submit your manuscripts at http://www.hindawi.com
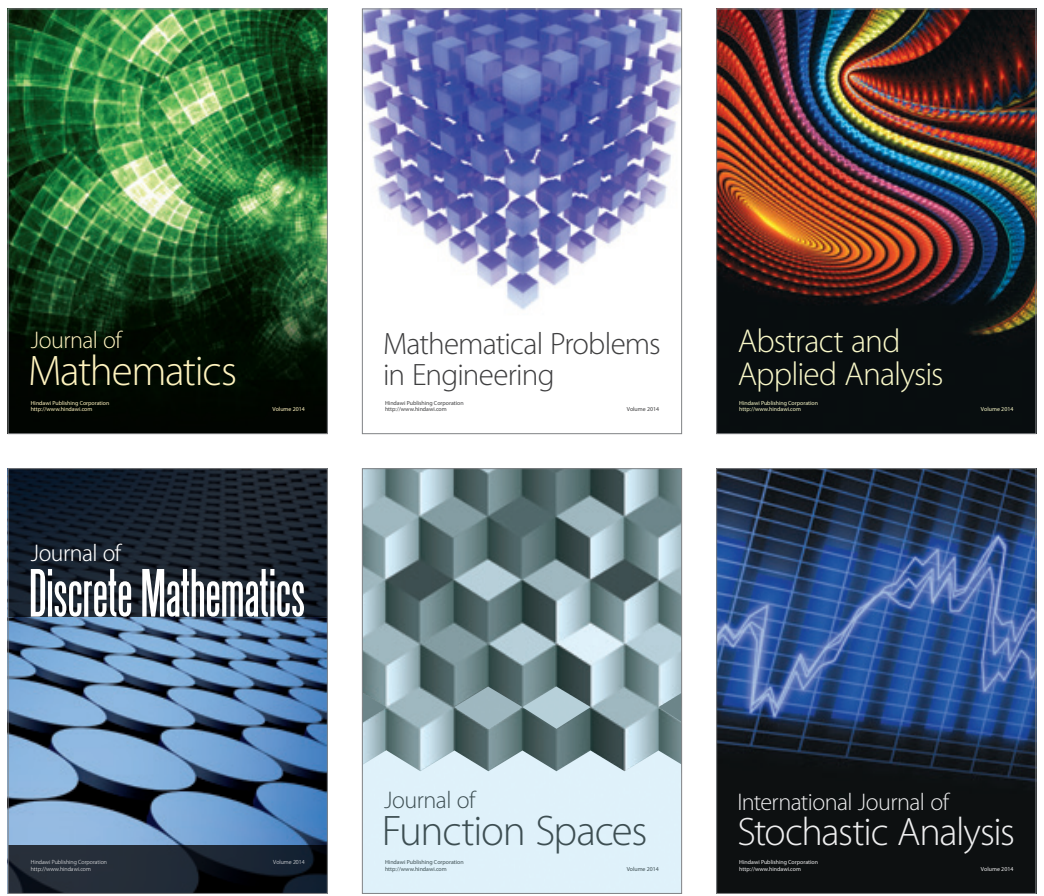

Journal of

Function Spaces

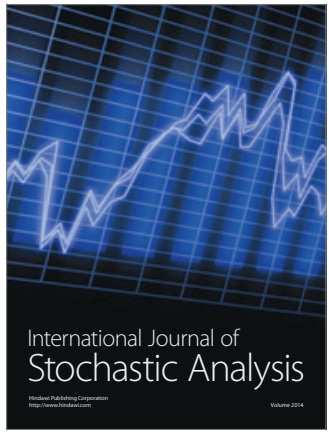

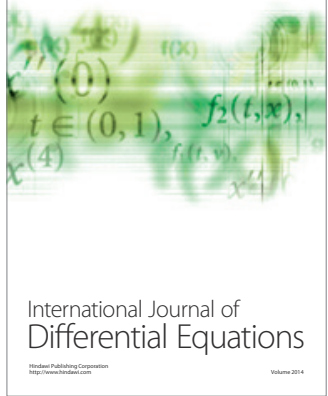
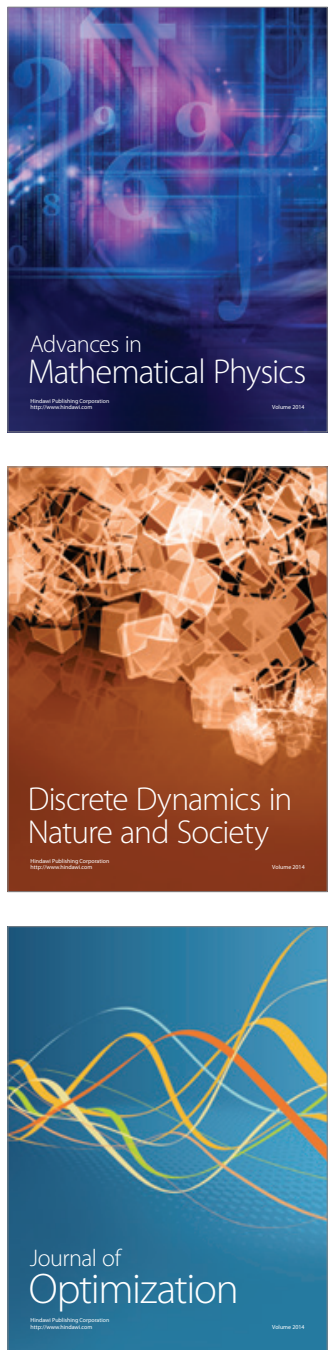